\title{
The Alloys of Aluminium and Silicon.
}

THE very great interest at present being taken in the aluminium alloys containing from about ro per cent. to I3 per cent. of silicon, is shown by the fact that no less than three of the papers presented to the Institute of Metals at the recent meeting at Liège dealt with this material.

Both from the practical point of view, regarding the material as one of considerable promise for the production of light alloy castings, and from the theoretical, these alloys are of more than usual interest. It has been known for some time that the structure may be greatly affected by the addition to the molten metal of small amounts of various 'modifying' reagents. Many theories concerning the reason for this change of structure have been propounded, but, hitherto, no completely satisfactory hypothesis has been available. Figs. I and 2, reproduced by courtesy of the Institute of Metals,

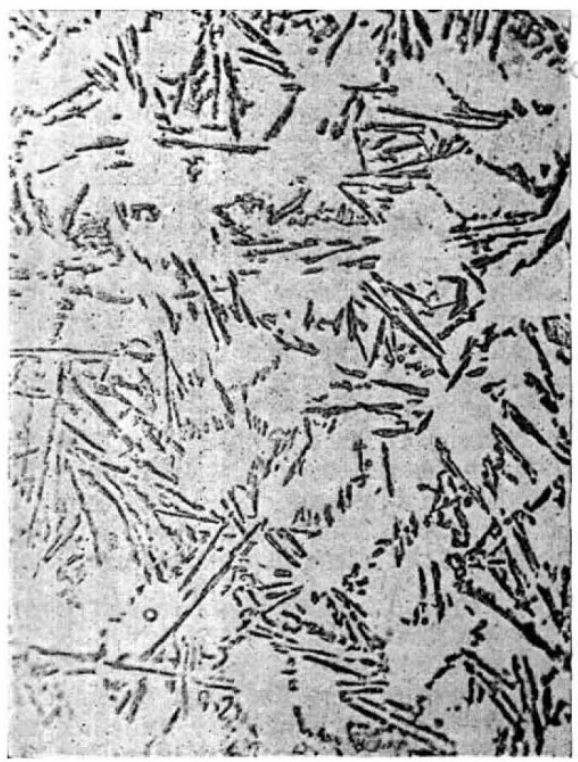

FrG. I. $-9 \cdot 86$ per cent. Si, $0 * 45$ per cent. Fe. Chill cast. Unetched. $\times 200$.

illustrate the change in structure of the Io per cent. alloy brought about by the addition, at $750^{\circ} \mathrm{C}$., of 5 per cent. of sodium hydroxide. Fig. I represents the material in the ordinary chill cast state, and Fig. 2 the same after the 'modifying' treatment. The mechanical properties also are greatly improved when the alloy possesses the second type of structure.

Of the three papers now before us, that by Dr. A. G. C. Gwyer and Mr. H. W. L. Phillips (with an appendix by Dr. Stockdale and Mr. I. Wilkinson which is concerned with the mechanical properties of the alloys) is the most important. The properties of the material in the 'normal' condition are not very remarkable, but the addition of a small amount of a suitable metal or salt to the melt before it is poured endows the casting with far superior strength and ductility.

In the normal state these alloys consist of a simple eutectiferous system, with small limits of solid solubility at each end. The thermal-equilibrium diagram has been redetermined, and the eutectic point placed at II.7 per cent. of silicon and at a temperature of $577^{\circ} \mathrm{C}$. After 'modificatior' the eutectic contains an appreciably greater amount of silicon and is formed at a lower temperature, both the composition and the temperature being dependent on the rate of cooling and the treatment of the metal. Further, when the molten metal is stirred or remelted after 'modification,' or left in the molten state for an undue length of time after the addition of the modifying material, the normal structure and properties are obtained.

There are clear indications, therefore, that the ' modified ' metal is in an unstable state. The authors mentioned now offer a new explanation of their own, which appears to approach far more nearly to the truth than anything which has yet been suggested.

This theory may be briefly stated as follows: When the aluminium-silicon alloy is raised to a temperature considerably higher than its melting point, it is probable that the silicon and aluminium are in true solution. When solid the greater portion

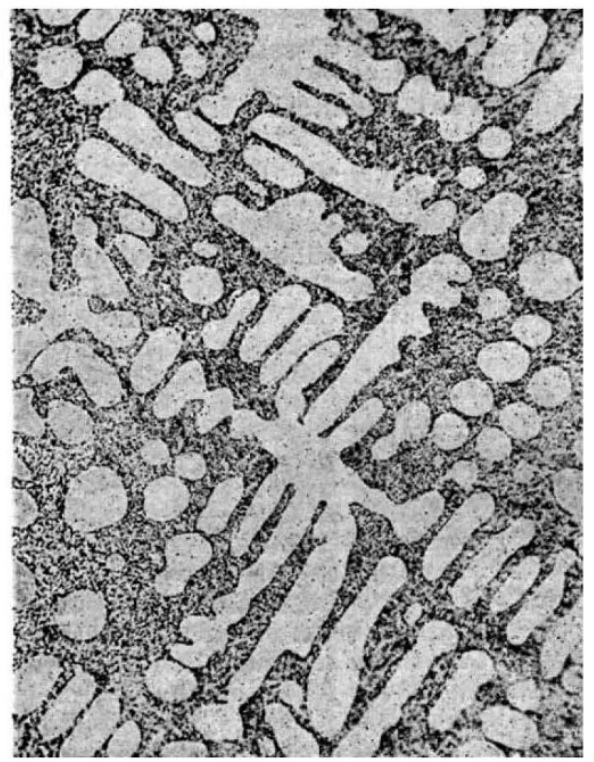

FIG. 2.- $9 \cdot 86$ per cent. Si, 0.45 per cent. Fe. Same alloy as Fig. I, but modified with 5 per cent. NaOH at $750^{\circ} \mathrm{C}$. Chill cast. Etch HF. $\times 200$.

of the silicon is present, dispersed in crystals of a size readily visible under the microscope. A small quantity, of the order of 0.5 per cent. at room temperature, remains in solid solution in the aluminium, but this may be disregarded at the moment. In solidifying, the silicon and aluminium pass from atomic dispersion to the crystalline form. At some stage during the process, therefore, the silicon and aluminium aggregates will be of colloidal dimensions. It is well known that the colloidal state is an unstable one, and that colloid particles tend to coalesce. Their rate of growth may be accelerated or diminished by the addition of protective agents.

Having regard to all the facts which have come to light, it appears to the authors that the most satisfactory explanation of the behaviour of 'modifying agents' is to assume that they function as colloid protectors. They do not, of course, confer complete protection upon the colloidal silicon and aluminium, but simply retard their aggregation. From the changes in the crystalline form of the constituents brought about by the process of 'modification,' it is probable that the protector itself is a colloid. It does not, however, appear that the assumption is 
justified that when sodium compounds are used for 'modifying,' the protector is necessarily metallic sodium. As a result of this action, the formation of the normal eutectic is inhibited, and aluminium continues to be precipitated from the metal until the super-solubility line is reached.

It is also shown that the protection of the modified structure can be obtained without the use of the ordinary reagents provided that the rate of cooling is sufficiently rapid.

From the theory that certain substances hinder the aggregation of the colloid particles, it should be possible to discover also other substances capable of accelerating this aggregation. The compound $\mathrm{FeAl}_{3}$ is such a substance, and it has been experimentally substantiated that the presence of iron in melts of these silicon-aluminium alloys is detrimental.

A diagram has been drawn up for the 'modified' alloys indicating the manner in which under-cooling alters the normal process of solidification. The amount of the substances added to effect the "modi- fication' has been considered, and with sodium hydroxide, and for an alloy of about I2 per cent. of silicon, it is put at about 5 per cent. of the weight of the metal. Excess results in a deterioration of the mechanical properties.

The time which is allowed to elapse between the addition of the castings is also of importance, and, for the usual composition of alloy, should be about half an hour.

Since the general theory propounded is independent of any special constituent, it should be sufficiently general to apply to systems other than that imme. diately under consideration. This is shown to be the case, and exactly analogous results have been obtained in the systems copper-aluminium, aluminiumnickel, aluminium-manganese, lead-antimony, and copper-antimony.

The importance of the colloidal state in alloys at the moment of solidification, and of under-cooling, is thus shown to be far greater than has hitherto been generally appreciated.
F. C. T.

\section{The Twelfth International Physiological Congress in Stockholm.}

$\mathrm{F}$ ACH international congress, as a rule, discloses some special interest in one or another field of physiclogical science, as well as serving as a sure guide to the virility of the science in general. Contrasted with the last congress, held three years ago in Edinburgh, the present congress had no one central interest comparable to that excited by the isolation of insulin, but the fine attendance of the foremost representatives, with a considerable number of the younger workers, from all the continental European countries, was a welcome evidence of the general recovery of physiology from the devastating effects of the War, and of the steady and persistent advance in almost every branch of this science.

The congress in Stockholm was as beautifully arranged as in Edinburgh, and as regards the welcome and hospitality offered there is no need to say more than that it could not have been bettered. The scientific programme of the congress opened with an address by Sir Frederick Gowland Hopkins on "The Mechanisms of Biological Oxidations," in which he outlined the position attained by workers in this field, with especial reference to the work done in his own laboratory at Cambridge.

The routine work of the congress was divided into four sections which were grouped according to subjects, facilitating the attendance by each member at the discussions on those papers in which he was particularly interested.

Great interest was aroused by a number of papers dealing with the fundamental processes of life, as, for example, the communications of the recent important researches of Adrian upon the afferent impulses conducted by the single nerve fibre, and of Hill and Downing upon the measurement of heat production in nerve. An interesting summary was given by Kato (Tokyo) of his theory of decrementless conduction in the narcotised region of nerve, in which, as is well known, he is opposed to the findings of Keith Lucas and Adrian. The problem was the subject of a lively debate, and is probably now near to solution. Muscle physiology was represented by Embden, by Hill, and by Meyerhof.

Another point of great interest was the question with regard to tissue metabolism, mainly represented by Dale's school, in relation to insulin and to such problems as the development of rigor.

In the realm of the central nervous system, an interesting communication was made by Samojloff
(Kasan) upon the nature of spinal inhibition. His experiments tend to support the theory of a liberation of some chemical substance affecting the synopses or the cellular structures in the spinal cord. Magnus (Utrecht) made a further contribution to the subject of local and segmental reflexes in the decerebrated animal.

Metabolism was represented by Lusk and Benedict, Boothby, Knoop, Mendel, Noyons, and de Barenne. A noteworthy tendency lay in the simplification of technique, and the applicability of the new methods to clinical practice. A summary was given by Mann (Rochester, Minn.) of his work on the dehepatised animal. Interest in the question of vitamins seems recently to have diminished, very few communications being made.

The study of digestion has now definitely shifted out of the hands of Pavlov's school to America, from whence the pupils of Carlsson, and particularly Ivy, brought communications dealing with the phases of gastric and pancreatic secretion.

Circulation was represented from America by Wiggers (Cleveland), who discussed the fractionate nature of ventricular contraction. The British school was represented by Anrep, who gave papers upon the central and reflex regulation of the coronary circulation (mainly in regard to the innervation of the coronary blood vessels), and upon the electrical measurement of coronary circulation during the single cardiac cycle. Heymans (Ghent) communicated the results obtained with his method of cerebral perfusion as applied to the study of the respiratory and cardioinhibitory centres. Frédéricq (Liège) summarised the results of his recent work upon chronaxie, especially in relation to the influence of the vagus and sympathetic cardiac nerves.

Prof. Loewi (Graz), in one of the most interesting papers of the congress, gave an account of the various factors affecting the rate of destruction of the chemical substance determining the inhibitory action of the vagus upon the heart. The work of this school would seem to bring us one step nearer to comprehension of the ultimate mechanism of peripheral inhibition.

An important contribution to the problem of hæmophilia was made by Howell (Baltimore), who brings evidence that the determining factor in this condition lies in an unusual stability of the blood platelets. In a second communication, Howell discussed the chemical nature of the anti-coagulant NO. 2970, VOL. I I 8$]$ 